\title{
Decentralised stabilising controllers for a class of large-scale linear systems
}

\author{
B C JHA ${ }^{1}, \mathrm{~K}_{\text {PATRALEKH }}^{2}$ and R SINGH ${ }^{2}$ \\ ${ }^{1}$ University Science Instrumentation Centre, T M Bhagalpur University, \\ Bhagalpur 812007 , India \\ ${ }^{2}$ Department of Electrical Engineering, Bhagalpur College of Engineering, \\ P O Sabour, Bhagalpur 813210 , India \\ e-mail: patralekhkameshwar@usa.net
}

MS received 30 October 1999

\begin{abstract}
A simple method for computing decentralised stabilising controllers for a class of large-scale (interconnected) linear systems has been developed. Decentralised controls are optimal controls at subsystem level and are generated from the solution of algebraic Riccati equations for decoupled subsystems resulting from a new aggregation-decomposition technique. The method has been illustrated through a numerical example of a large-scale linear system consisting of three subsystems each of the fourth order.
\end{abstract}

Keywords. Decentralised stabilisation; large-scale linear systems; optimal feedback control; algebraic Riccati equations.

\section{Introduction}

Various recent investigators (Wang \& Davison 1973; Siljak \& Vukcevic 1977; Mahalanabis \& Singh 1980) have studied the problems of decentralised stabilisation of large-scale (interconnected) linear time-invariant systems. The decentralised stabilisation methods suggested by Wang \& Davison (1973) and Siljak \& Vukcevic (1977) are based on the centralised stability condition and that by Mahalanabis \& Singh (1980) on the decentralised stability condition. Wang \& Davison (1973) have proposed a computer algorithm for determining the decentralised feedback compensator parameters, which would place the eigenvalues of the compensated composite system at the desired locations. For a large-scale system consisting of $N$ subsystems each of order $n_{i}$, the proposed technique requires trial and error runs of eigenvalues computation subroutines for the entire system of order $N \times n_{i}$. Siljak \& Vukcevik (1977) obtain the comparison system using the vector Liapunov function-based aggregation technique. The desired stabilising local control gain vector is then computed by applying determinantal inequality tests to the aggregate matrix of the comparison system. Hence, the methods of Wang \& Davison (1973) and Siljak \&Vukcevic (1977) are computationally very involved. Mahalanabis \& Singh (1980) have suggested a 
method for computing decentralised stabilising controllers by utilising the decentralised stability condition established by the scalar Liapunov function-based aggregation-decomposition technique. But this method also is computationally not attractive. Moreover, the results of all the work discussed above are conservative.

The aim of the present work is to develop a computationally simple and less conservative method of decentralised stabilisation for the same class of linear interconnected systems as discussed by Siljak \& Vukcevic (1977) and Mahalanabis \& Singh (1980). It is further desired that the decentralised stabilisation is achieved with optimal response and minimum expense of control energy. In order to achieve this, first the effects of interactions are incorporated into the coefficient matrices of the interaction-free parts resulting in decoupled subsystems by a new aggregation-decomposition technique, which is based on algebraic Riccati equations for the interaction-free parts. Desired decentralised stabilising controllers are computed from the solution of the algebraic Riccati equations for the resulting decoupled subsystems. Since the algebraic Riccati equations result from the optimal control problem for the linear time-invariant (decoupled) subsystems with quadratic performance criteria, the decentralised controllers stabilise the system with optimal response and minimum expense of control energy and are, therefore, the best decentralised stabilising controllers.

\section{Problem formulation}

Consider a large-scale linear time-invariant system represented as $N$ linear interconnected subsystems as in Siljak \& Vukcevik (1977) and Mahalanabis \& Singh (1980). The state equations of the $i$ th subsystem have the form:

$$
\dot{x}_{i}=A_{i i} x_{i}+b_{i} u_{i}+\sum_{j=1, j \neq i}^{N} A_{i j} x_{j}, \quad i=1,2, \ldots, N,
$$

where the state vector $x_{i} \triangleq\left[x_{i 1} x_{i 2} \ldots x_{i n_{i}}\right]^{T}$ and $u_{i}$ is the scalar control. $A_{i i}$ is the coefficient matrix of the interaction-free part and $b_{i}$ is the driving vector. It is assumed that $\left(A_{i i}, b_{i}\right)$ is in companion form,

$$
A_{i i}\left[\begin{array}{cccccc}
0 & 1 & 0 & 0 & \cdots & 0 \\
0 & 0 & 1 & 0 & \cdots & 0 \\
\cdots & \cdots & \cdots & \cdots & \cdots & \cdots \\
\cdots & \cdots & \cdots & \cdots & \cdots & \cdots \\
0 & 0 & 0 & \cdots & 0 & 1 \\
-c_{i 1} & -c_{i 2} & -c_{i 3} & \cdots & \cdots & -c_{i n_{i}}
\end{array}\right], \quad b_{i}=[0,0,0, \ldots, 1]^{T} .
$$

It is further assumed that $\left(A_{i i}, b_{i}\right)$ is completely controllable. The term $\sum_{j=1, j \neq 1}^{N} A_{i j} x_{j}$ represents the interaction among the $i$ th and other subsystems. $A_{i j}$ is an $n_{i} \times n_{j}$ real matrix. The problem to be studied is the determination of the decentralised state feedback controller gain vector $K_{i}$ for generating decentralised feedback control,

$$
u_{i}=K_{i} x_{i}, i=1,2, \ldots, N,
$$

for each of the $i$ th subsystem of equation (1) such that the composite system is stable. It is further desired that the $i$ th subsystem is stabilised with optimal response and the cost of the control energy is minimum. 


\section{Aggregation-decomposition}

In order to incorporate the effects of interactions into the coefficient matrix of the interaction-free part of the $i$ th subsystem of (1), a quadratic function $V_{i}\left(x_{i}\right)$ is considered such that

$$
V_{i}\left(x_{i}\right)=x_{i}^{T} R_{i} x_{i}
$$

where $R_{i}$ is an $n_{i} \times n_{i}$ real symmetric positive definite matrix obtained as the solution of the algebraic Riccati equation,

$$
\left(1 / \lambda_{i}\right) R_{i} b_{i} b_{i}^{T} R_{i}=A_{i i}^{T} R_{i}+R_{i} A_{i i}+Q_{i},
$$

for the interaction-free part of the $i$ th subsystem of (1). This implies that $V_{i}\left(x_{i}\right)$ is positive definite. $Q_{i}$ in (3) is an $n_{i} \times n_{i}$ real symmetric positive definite matrix and $\lambda_{i}$ is a positive constant. It then follows that for the interaction-free part of the $i$ th subsystem of (1)

$$
x_{i}^{T}\left(\left(1 / \lambda_{i}\right) R_{i} b_{i} b_{i}^{T} R_{i}\right) x_{i}=x_{i}^{T}\left(A_{i i}^{T} R_{i}+R_{i} A_{i i}\right) x_{i}+x_{i}^{T} Q_{i} x_{i} .
$$

It is to be noted that (appendix A):

$$
x_{i}^{T}\left(A_{i i}^{T} R_{i}+R_{i} A_{i i}\right) x_{i}=\left[\operatorname{grad} V_{i}\left(x_{i}\right)\right]^{T} A_{i i} x_{i} .
$$

Hence, for the $N$ interconnected subsystems of (1), it follows that (appendix B):

$$
\begin{aligned}
\sum_{i=1}^{N} x_{i}^{T}\left(\frac{1}{\lambda_{i}} R_{i} b_{i} b_{i}^{T} R_{i}\right) x_{i}=\sum_{i=1}^{N} & {\left[\operatorname{grad} V_{i}\left(x_{i}\right)\right]^{T} A_{i i} x_{i} } \\
& +\sum_{i=1}^{N} \sum_{j=1, j \neq i}^{N}\left[\operatorname{grad} V_{i}\left(x_{i}\right)\right]^{T} A_{i j} x_{j}+\sum_{i=1}^{N} x_{i}^{T} Q_{i} x_{i} .
\end{aligned}
$$

As shown in appendix C:

$$
\sum_{i=1}^{N} \sum_{j=1, j \neq i}^{N}\left[\operatorname{grad} V_{i}\left(x_{i}\right)\right]^{T} A_{i j} x_{j} \leq \sum_{i=1}^{N} x_{i}^{T} M_{i} x_{i}
$$

where $M_{i}$ is an $n_{i} \times n_{i}$ real positive definite diagonal matrix. Using the above inequality, (4) is converted to the following inequality (appendix D):

$$
\begin{aligned}
\sum_{i=1}^{N} x_{i}^{T}\left(\frac{1}{\lambda_{i}} R_{i} b_{i} b_{i}^{T} R_{i}\right) x_{i} & \leq \sum_{i=1}^{N} x_{i}^{T}\left(A_{i i}^{T} R_{i}+R_{i} A_{i i}\right) x_{i} \\
& +\sum_{i=1}^{N} 2 m_{i} x_{i}^{T} R_{i} x_{i}+\sum_{i=1}^{N} x_{i}^{T} Q_{i} x_{i}
\end{aligned}
$$

where,

case (i):

$$
m_{i}=1 /\left(2 \beta_{i \max }\right),
$$


$\beta_{i \max }$ being the maximum eigenvalue of the real symmetric positive definite matrix $S_{i}$ given by,

$$
S_{i}=G_{i}^{-1} R_{i}\left(G_{i}^{-1}\right)^{T}, \quad G_{i} G_{i}^{T}=M_{i},
$$

when the eigenvalues of $S_{i}$ are all real and positive which implies that $m_{i}$ is a real and positive number, or

$$
m_{i}=1 /\left(2 \beta_{i \min }\right),
$$

$\beta_{i \text { min }}$ being the minimum eigenvalue of $S_{i}$ as defined above, when the eigenvalues of $S_{i}$ are all real and the minimum eigenvalue is negative which implies that $m_{i}$ is a real and negative number.

Case (ii): When $S_{i}$ has at least one pair of complex eigenvalues, $m_{i}$ is given as

$$
m_{i}=1 /\left(2 \bar{a}_{i \max } / a_{i \min }\right),
$$

$a_{i \text { min }}$ being the lowest diagonal element of $M_{i}$ and $\bar{a}_{i \text { max }}$ being the highest diagonal element of another real positive definite diagonal matrix $\overline{\mathbf{M}}_{i}$ such that

$$
x_{i}^{T} R_{i} x_{i} \leq x_{i}^{T} \bar{M}_{i} x_{i}
$$

$m_{i}$ is a real and positive number.

The inequality (6) can be replaced by an equation, by replacing $R_{i}$ by another $n_{i} \times n_{i}$ real symmetric positive definite matrix $\mathbf{R}_{i}$.

$$
\begin{aligned}
\sum_{i=1}^{N} x_{i}^{T}\left(\frac{1}{\lambda_{i}} \mathbf{R}_{i} b_{i} b_{i}^{T} \mathbf{R}_{i}\right) x_{i}=\sum_{i=1}^{N} x_{i}^{T} & \left(A_{i i}^{T} \mathbf{R}_{i}+\mathbf{R}_{i} A_{i i}\right) x_{i} \\
& +\sum_{i=1}^{N} 2 m_{i} x_{i}^{T} \mathbf{R}_{i} x_{i}+\sum_{i=1}^{N} x_{i}^{T} Q_{i} x_{i},
\end{aligned}
$$

which is reduced to the following equation (appendix E):

$$
\left(1 / \lambda_{i}\right) \mathbf{R}_{i} b_{i} b_{i}^{T} \mathbf{R}_{i}=\left(A_{i i}+m_{i} I\right)^{T} \mathbf{R}_{i}+\mathbf{R}_{i}\left(A_{i i}+m_{i} I\right)+Q_{i},
$$

which is the algebraic Riccati equation for the system:

$$
\dot{x}_{i}=\left(A_{i i}+m_{i} I\right) x_{i}+b_{i} u_{i}, \quad i=1,2, \ldots, N .
$$

If (12) is compared with (1), it is observed that the effects of interactions have been incorporated (aggregated) as ' $m_{i} I$ ' into the coefficient matrix of the $i$ th subsystem, so that $N$ interconnected subsystems of (1) have been decomposed into $N$ decoupled subsystems of (12). The real number $m_{i}$ is, therefore, designated the interaction coefficient and the procedure is called aggregation-decomposition.

\section{Decentralised stabilisation by optimal feedback controls at subsystems level}

Equations (12) and (11) can be rewritten as

$$
\dot{x}_{i}=A_{m i} x_{i}+b_{i} u_{i}, \quad i=1,2, \ldots, N,
$$


and

$$
\left(1 / \lambda_{i}\right) \mathbf{R}_{i} b_{i} b_{i}^{T} \mathbf{R}_{i}=A_{m i}^{T} \mathbf{R}_{i}+\mathbf{R}_{i} A_{m i}+Q_{i},
$$

where

$$
A_{m i} \triangleq A_{i i}+m_{i} I
$$

$A_{m i}$ is the modified coefficient matrix of the $i$ th subsystem incorporating the maximum possible interaction effects. Hence, decentralised stabilisation of interconnected subsystem of (1) implies the stabilisation of the decoupled subsystems of (13).

It is noted that the interaction-free part of the $i$ th interconnected subsystem of (1) is in controllable phase variable form. The $i$ th decoupled subsystem of (13) has the same $b_{i}$ as in (1), but $A_{m i}$ is not in controllable phase variable form. Hence, a similarity transformation (Kuo 1993) with $n_{i} \times n_{i}$ transformation matrices $P_{i}$ is applied to the decoupled subsystems of (13). The resulting $i$ th transformed decoupled subsystem in phase variable form is written as

$$
\dot{\bar{x}}_{i}=\bar{A}_{m i} \bar{x}_{i}+b_{i} u_{i},
$$

where

$$
\bar{x}_{i} \triangleq\left[\begin{array}{lllll}
\bar{x}_{i 1} & \bar{x}_{i 2} & \cdots & \bar{x}_{i n_{i}}
\end{array}\right]^{T} \text {. }
$$

Then the algebraic Riccati equation (14) is rewritten as

$$
\left(1 / \lambda_{i}\right) \bar{R}_{i} b_{i} b_{i}^{T} \bar{R}_{i}=\bar{A}_{m i}^{T} \bar{R}_{i}+\bar{R}_{i} \bar{A}_{m i}+Q_{i},
$$

in (15) and (16), $\left(\bar{A}_{m i}, b_{i}\right)$ is in companion form, $\bar{K}_{i}$, given by

$$
\bar{K}_{i}=-\left(1 / \lambda_{i}\right) b_{i}^{T} \bar{R}_{i}
$$

is the optimal controller gain for the $i$ th decoupled subsystem of (15), $\bar{R}_{i}$ again being an $n_{i} \times n_{i}$ real symmetric positive definite matrix, given as the solution of the algebraic Riccati equation (16), resulting from the optimal control problem for the linear timeinvariant decoupled $i$ th subsystem of (15) with quadratic performance criterion:

$$
I=\int_{0}^{\infty}\left[x_{i}^{T} Q_{i} x_{i}+\lambda_{i} u_{i}^{2}\right] \mathrm{d} t .
$$

Since the optimal control function $u_{i}^{*}$, given by

$$
u_{i}^{*}=\bar{K}_{i} x_{i}
$$

minimises the quadratic performance criterion, the $i$ th decoupled subsystem of (15) is stabilised with optimal response at minimum cost of control energy.

For the $i$ th decoupled subsystem (13), the optimal controller gain $K_{i}$ is given by:

$$
K_{i}=\bar{K}_{i} P_{i}
$$

It then follows that $K_{i}$ given by (19) is the desired decentralised stabilising controller gain to generate the decentralised control function $u_{i}$ as per (2) which guarantees the stability of the $i$ th interconnected subsystem (1) with optimal response and minimum cost of control energy. However, the result is slightly conservative because of majorisation in aggregationdecomposition. $K_{i}$ is, therefore, the best possible decentralised stabilising controller gain. 


\section{Solution of the algebraic Riccati equations}

As already stated, aggregation-decomposition and the decentralised stabilisation by optimal feedback controls at subsystems level require the solution of the algebraic Riccati equations (3) and (16). Since $\left(A_{i i}, b_{i}\right)$ in (3) and $\left(\bar{A}_{m i}, b_{i}\right)$ in (16) are both in companion form, the computationally simple method of solution reported in the literature (Singh 1970) can be directly applied. The method requires the solution of only $\left(n_{i}-1\right)$ nonlinear algebraic equations by Newton-Raphson method for subsystems of order $n_{i}$.

\section{Numerical example}

Three linear interconnected fourth-order subsystems corresponding to (1) are considered:

$$
\begin{aligned}
& \dot{x}_{1}=\left[\begin{array}{cccc}
0 & 1 & 0 & 0 \\
0 & 0 & 1 & 0 \\
0 & 0 & 0 & 1 \\
0 & -6 & -11 & -6
\end{array}\right] x_{1}+\left[\begin{array}{l}
0 \\
0 \\
0 \\
1
\end{array}\right] u_{1}+\left[\begin{array}{cccc}
0 & 0 & 0 & 0 \\
0 & 0 & 0 & 0 \\
0 & 0 & 0 & 0 \\
0 & 0.05 & 0 & 0
\end{array}\right] x_{2} \\
& \\
& +
\end{aligned}
$$

With $Q_{1}=Q_{2}=Q_{3}=I_{4}$ and $\lambda_{1}=\lambda_{2}=\lambda_{3}=+1$ in the algebraic Riccati equations corresponding to (3), the values of $R_{1}, R_{2}, R_{3}$ for the subsystems are obtained. The values of $M_{1}, M_{2}, M_{3}$ corresponding to inequality (5) are computed using the above values of $R_{1}, R_{2}, R_{3}$. Then $S_{1}, S_{2}, S_{3}$ are computed using (8). 
Since the eigenvalues of $S_{1}$ are all real and positive, using (7), one gets $m_{1}=1$ / $\left(2 \beta_{1 \max }\right)=0.1$ and as the minimum eigenvalues of $S_{2}$ and $S_{3}$ are negative, using (9), one gets $m_{2}=1 /\left(2 \beta_{2 \mathrm{~min}}\right)=-0.6328, m_{3}=1 /\left(2 \beta_{3 \mathrm{~min}}\right)=-0.091$. Hence, the three decoupled subsystems corresponding to (13) are obtained. The decoupled subsystems on being transformed to controllable phase variable form corresponding to (15) with transformation matrices $P_{1}, P_{2}, P_{3}$ become:

$$
\begin{aligned}
\dot{\bar{x}}_{1}= & {\left[\begin{array}{cccc}
0 & 1 & 0 & 0 \\
0 & 0 & 1 & 0 \\
0 & 0 & 0 & 1 \\
0.5395 & -1.2959 & -5.9417 & -4.7772
\end{array}\right] \bar{x}_{1}+\left[\begin{array}{l}
0 \\
0 \\
0 \\
1
\end{array}\right] u_{1}, } \\
\dot{\bar{x}}_{2}= & {\left[\begin{array}{cccc}
0 & 1 & 0 & 0 \\
0 & 0 & 1 & 0 \\
0 & 0 & 0 & 1 \\
-6.1567 & -15.0547 & -14.603 & -6.4642
\end{array}\right] \bar{x}_{2}+\left[\begin{array}{l}
0 \\
0 \\
0 \\
1
\end{array}\right] u_{2}, } \\
\dot{\bar{x}}_{3}= & {\left[\begin{array}{cccc}
0 & 1 & 0 & 0 \\
0 & 0 & 1 & 0 \\
0 & 0 & 0 & 1 \\
-1.7596 & -9.2899 & -10.9112 & -6.1570
\end{array}\right] \bar{x}_{3}+\left[\begin{array}{l}
0 \\
0 \\
0 \\
1
\end{array}\right] u_{3} . }
\end{aligned}
$$

Then $\bar{R}_{1}, \bar{R}_{2}, \bar{R}_{3}$ are computed by solving (16). Hence, decentralised stabilising controller gains $\bar{K}_{1}, \bar{K}_{2}, \bar{K}_{3}$ are computed by using (17). Finally, corresponding to (19), the desired decentralised stabilising controller gains are computed as

$$
\begin{aligned}
& K_{1}=\left[\begin{array}{llll}
-1.0467 & -2.6256 & -1.1798 & -0.2843
\end{array}\right], \\
& K_{2}=\left[\begin{array}{llll}
-0.7441 & -2.6356 & -1.6794 & -0.4029
\end{array}\right], \\
& K_{3}=\left[\begin{array}{llll}
-0.9605 & -13.3048 & -8.1428 & -1.7551
\end{array}\right] .
\end{aligned}
$$

\section{Conclusions}

The method of decentralised stabilisation of linear interconnected systems by optimal feedback controls at subsystems level as suggested in the present work is entirely new. The method is based on aggregation-decomposition technique which is based on algebraic Riccati equations, whereas the aggregation-decomposition technique suggested by earlier authors is based on Liapunov functions. The decentralised stabilisation procedure suggested in the present work is computationally simpler because it requires the solution of only $\left(n_{i}-1\right)$ nonlinear algebraic equations for the subsystems of order $n_{i}$. The method suggested in the literature (Mahalanabis \& Singh 1980) requires the solution of $n_{i}\left(n_{i}+1\right) / 2$ Liapunov equations for subsystems of order $n_{i}$. These results are less conservative than the earlier results. Since the decentralised controls are optimal feedback controls which minimise the quadratic performance criteria, the subsystems are stabilised with optimal response and minimum expenditure of control energy. The result of the present work is slightly conservative due to majorisation in aggregation-decomposition. Since the procedure can be programmed on a digital computer, the computation of the gain parameters for decentralised stabilising controllers can be carried out conveniently even for subsystems of higher order. 
Implementation of the controls is quite cheap and simple, because it requires only amplifiers and coefficient multipliers. Performance of the compensated system can be foreseen by simulation.

The proposed method can be extended to bilinear, nonlinear and stochastic interconnected systems.

\section{Appendix A.}

Since $V_{i}\left(x_{i}\right)=x_{i}^{T} R_{i} x_{i}$, where $R_{i}$ is a real symmetric positive definite matrix,

$$
\left[\operatorname{grad} V_{i}\left(x_{i}\right)\right]^{T} A_{i i} x_{i}=2 x_{i}^{T} R_{i} A_{i i} x_{i}=x_{i}^{T} R_{i} A_{i i} x_{i}+x_{i}^{T} R_{i} A_{i i} x_{i} .
$$

Noting that $x_{i}^{T} R_{i} A_{i i} x_{i}$ is a scalar,

$$
\begin{aligned}
x_{i}^{T} R_{i} A_{i i} x_{i} & =\left[x_{i}^{T} R_{i} A_{i i} x_{i}\right]^{T}=x_{i}^{T} A_{i i}^{T} R_{i} x_{i}, \\
x_{i}^{T} A_{i i}^{T} R_{i} x_{i}+x_{i}^{T} R_{i} A_{i i} x_{i} & =\left[\operatorname{grad} V_{i}\left(x_{i}\right)\right]^{T} A_{i i} x_{i}, \\
x_{i}^{T}\left(A_{i i}^{T} R_{i}+R_{i} A_{i i}\right) x_{i} & =\left[\operatorname{grad} V_{i}\left(x_{i}\right)\right]^{T} A_{i i} x_{i} .
\end{aligned}
$$

\section{Appendix B.}

For the interaction-free part of the ith subsystem of (1),

$$
x_{i}^{T}\left(\left(1 / \lambda_{i}\right) R_{i} b_{i} b_{i}^{T} R_{i}\right) x_{i}=\left[\operatorname{grad} V_{i}\left(x_{i}\right)\right]^{T} A_{i i} x_{i}+x_{i}^{T} Q_{i} x_{i},
$$

For the ith interconnected subsystem of (1),

$$
\begin{aligned}
& x_{i}^{T}\left(\left(1 / \lambda_{i}\right) R_{i} b_{i} b_{i}^{T} R_{i}\right) x_{i}=\left[\operatorname{grad} V_{i}\left(x_{i}\right)\right]^{T}\left(A_{i i} x_{i}+\sum_{j=1, j \neq i}^{N} A_{i j} x_{j}\right)+x_{i}^{T} Q_{i} x_{i}, \\
& x_{i}^{T}\left(\left(1 / \lambda_{i}\right) R_{i} b_{i} b_{i}^{T} R_{i}\right) x_{i}=\left[\operatorname{grad} V_{i}\left(x_{i}\right)\right]^{T} A_{i i} x_{i}+\sum_{j=1, j \neq i}^{N}\left[\operatorname{grad} V_{i}\left(x_{i}\right)\right]^{T} A_{i j} x_{j}+x_{i}^{T} Q_{i} x_{i} .
\end{aligned}
$$

Hence for $N$ interconnected subsystems it follows that:

$$
\begin{aligned}
\sum_{i=1}^{N} x_{i}^{T}\left(\frac{1}{\lambda_{i}} R_{i} b_{i} b_{i}^{T} R_{i}\right) x_{i}=\sum_{i=1}^{N}\left[\operatorname{grad} V_{i}\left(x_{i}\right)\right]^{T} A_{i i} x_{i} \\
+\sum_{i=1}^{N} \sum_{j=1, j \neq 1}^{N}\left[\operatorname{grad} V_{i}\left(x_{i}\right)\right]^{T} A_{i j} x_{j}+\sum_{i=1}^{N} x_{i}^{T} Q_{i} x_{i} .
\end{aligned}
$$

\section{Appendix C.}

Noting that $V_{i}\left(x_{i}\right)=x_{i}^{T} R_{i} x_{i}$, where $R_{i}$ is a real symmetric positive definite matrix given by

$$
R_{i}=\left[\begin{array}{cccc}
r_{i 11} & r_{i 12} & \cdots & r_{i 1 n_{i}} \\
r_{i 12} & r_{i 22} & \cdots & r_{i 2 n_{i}} \\
\vdots & \vdots & \vdots & \vdots \\
r_{i 1 n_{i}} & r_{i 2 n_{i}} & \cdots & r_{i n_{i} n_{i}}
\end{array}\right]
$$


and

$$
\begin{aligned}
& A_{i j}=\left[\begin{array}{cccc}
a_{i j 11} & a_{i j 12} & \cdots & a_{i j 1 n_{j}} \\
a_{i j 21} & a_{i j 22} & \cdots & a_{i j 2 n_{j}} \\
\vdots & \vdots & \vdots & \vdots \\
a_{i j n_{i} 1} & a_{i j n_{i} 2} & \cdots & a_{i j n_{i} n_{j}}
\end{array}\right], \\
& {\left[\operatorname{grad} V_{i}\left(x_{i}\right)\right]^{T} A_{i j} x_{j}=\left[\frac{\partial V_{i}\left(x_{i}\right)}{\partial x_{i 1}} \frac{\partial V_{1}\left(x_{i}\right)}{\partial x_{i 2}} \cdots \frac{\partial V_{i}\left(x_{i}\right)}{\partial x_{i n i}}\right]} \\
& \times\left[\begin{array}{ccc}
a_{i j 11} x_{j 1}+a_{i j 12} x_{j 2}+\cdots+a_{i j 1 n j} x_{j n j} \\
a_{i j 21} x_{j 1}+a_{i j 22} x_{j 2}+\cdots+a_{i j 2 n j} x_{j n j} \\
\vdots & \vdots & \vdots \\
a_{i j n i 1} x_{j 1}+a_{i j n i 2} x_{j 2}+\cdots+a_{i j n i n j} x_{j n j}
\end{array}\right] .
\end{aligned}
$$

If the multiplications are carried out after explicitly evaluating the partial derivatives, terms of the form

$$
2\left(\sum_{w=1}^{n_{i}} r_{i w 1} a_{i j w w^{\prime}}\right) x_{i 1} x_{j w^{\prime}}
$$

$\left[i, j=1,2, \ldots, N ; w, 1=1,2, \ldots, n_{i} ; w^{\prime}=1,2, \ldots, n_{j}\right]$ are obtained, which can be bounded as below:

$$
2\left(\sum_{w=1}^{n_{i}} r_{i w 1} a_{i j w w^{\prime}}\right) x_{i 1} x_{j w^{\prime}} \leq\left|\sum_{w=1}^{n_{i}} r_{i w 1} a_{i j w w^{\prime}}\right|\left(x_{i 1}^{2}+x_{j w^{\prime}}^{2}\right),
$$

where $r_{i w 1}$ represents the $w, 1$ th element of the matrix $R_{i}$ and $a_{i j w w^{\prime}}$ the $w, w^{\prime}$ th element of the matrix $A_{i j}$.

Using this, it is then possible to obtain the inequality

$$
\left[\operatorname{grad} V_{i}\left(x_{i}\right)\right]^{T} A_{i j} x_{j} \leq x_{i}^{T} D_{i i}^{\prime} x_{i}+x_{j}^{T} D_{i j} x_{j},
$$

where $D_{i i}^{\prime}$ and $D_{i j}$ are diagonal matrices whose elements are real and non-negative numbers depending on the elements of the matrices $A_{i j}$ and $R_{i}$. It then follows that the following inequality must hold good:

$$
\begin{aligned}
\sum_{j=1, j \neq i}^{N}\left[\operatorname{grad} V_{i}\left(x_{i}\right)\right]^{T} A_{i j} x_{j} \leq \sum_{j=1, j \neq i}^{N}\left(x_{i}^{T} D_{i i}^{\prime} x_{i}+x_{j}^{T} D_{i j} x_{j}\right) & \\
& \times \sum_{j=1, j \neq i}^{N}\left[\operatorname{grad} V_{i}\left(x_{i}\right)\right]^{T} A_{i j} x_{j} \leq x_{i}^{T}\left\{(N-1) D_{i i}^{\prime}\right\} x_{i}+\sum_{j=1, j \neq i}^{N} x_{j}^{T} D_{i j} x_{j}, \\
& \times \sum_{j=1, j \neq i}^{N}\left[\operatorname{grad} V_{i}\left(x_{i}\right)\right]^{T} A_{i j} x_{j} \leq x_{i}^{T} D_{i i} x_{i}+\sum_{j=1, j \neq i}^{N} x_{j}^{T} D_{i j} x_{j},
\end{aligned}
$$

where $D_{i i}=(N-1) D_{i i}^{\prime}$. 


$$
\begin{aligned}
& \sum_{j=1, j \neq i}^{N}\left[\operatorname{grad} V_{i}\left(x_{i}\right)\right]^{T} A_{i j} x_{j} \\
& \leq \sum_{j=1}^{N} x_{j}^{T} D_{i j} x_{j} \sum_{i=1}^{N} \sum_{j=1, j \neq i}^{N}\left[\operatorname{grad} V_{i}\left(x_{i}\right)\right]^{T} A_{i j} x_{j} \\
& \leq \sum_{i=1}^{N} \sum_{j \neq 1}^{N} x_{j}^{T} D_{i j} x_{j} \sum_{i=1}^{N} \sum_{j=1, j \neq i}^{N}\left[\operatorname{grad} V_{i}\left(x_{i}\right)\right]^{T} A_{i j} x_{j} \\
& \leq \sum_{i=1}^{N} x_{i}^{T}\left(\sum_{j=1}^{N} D_{j i}\right) x_{j i} \sum_{i=1}^{N} \sum_{j=i, j \neq i}^{N}\left[\operatorname{grad} V_{i}\left(x_{i}\right)\right]^{T} A_{i j} x_{j} \\
& \leq \sum_{i=1}^{N} x_{i}^{T} M_{i} x_{i},
\end{aligned}
$$

where $M_{i}=\sum_{j=1}^{N} D_{j i}$.

\section{Appendix D.}

An $n_{i} \times n_{i}$ real positive definite diagonal (nonsingular) matrix $G_{i}$ is chosen such that

$$
G_{i} G_{i}^{T}=M_{i} .
$$

Then since $R_{i}$ is an $n_{i} \times n_{i}$ real symmetric positive definite matrix and $G_{i}$ and $G_{i}^{-1}$ are also $n_{i} \times n_{i}$ real positive definite diagonal (nonsingular) matrices, $S_{i}=G_{i}^{-1} R_{i}\left(G_{i}^{-1}\right)^{T}$ is also an $n_{i} \times n_{i}$ real symmetric positive definite matrix.

Case (i): When the eigenvalues of $S_{i}$ are all real and positive,

$$
I \geq\left(1 / \beta_{i \max }\right) S_{i},
$$

where $\beta_{i \max }$ is the maximum eigenvalue of $S_{i}$.

$$
\begin{aligned}
I & \geq\left(1 / \beta_{i \max }\right) G_{i}^{-1} R_{i}\left(G_{i}^{-1}\right)^{T}, \\
G_{i} G_{i}^{T} & \geq\left(1 / \beta_{i \max }\right) R_{i}, \\
M_{i} & \geq\left(1 / \beta_{i \max }\right) R_{i}, \\
x_{i}^{T} M_{i} x_{i} & \geq\left(1 / \beta_{i \max }\right)\left(x_{i}^{T} R_{i} x_{i}\right), \\
x_{i}^{T} M_{i} x_{i} & \geq 2 m_{i} x_{i}^{T} R_{i} x_{i},
\end{aligned}
$$

where $m_{i}=1 /\left(2 \beta_{i \max }\right)$ is a real and positive number, or when the eigenvalues of $S_{i}$ are all real but the minimum eigenvalue is negative,

$$
I \geq\left(1 / \beta_{i \min }\right) S_{i},
$$

where $S_{i}$ is as defined above, $\beta_{i \min }$ is the minimum eigenvalue of $S_{i}$ and is negative,

$$
I \geq\left(1 / \beta_{i \min }\right) G_{i}^{-1} R_{i}\left(G_{i}^{-1}\right)^{T} .
$$

Hence, proceeding as above, one gets

$$
x_{i}^{T} M_{i} x_{i} \geq 2 m_{i} x_{i}^{T} R_{i} x_{i},
$$

where $m_{i}=1 /\left(2 \beta_{i \min }\right)$ is a real and negative number. 
Case (ii): When $S_{i}$ has at least one pair of complex eigenvalues.

It is always possible to find a real positive definite diagonal matrix $\mathbf{M}_{i}$ such that

$$
\begin{aligned}
x_{i}^{T} R_{i} x_{i} & \leq x_{i}^{T} \mathbf{M}_{i} x_{i}, \\
\left(1 / \mathbf{a}_{i \max }\right) x_{i}^{T} R_{i} x_{i} & \leq\left(1 / \mathbf{a}_{i \max }\right) x_{i}^{T} \mathbf{M}_{i} x_{i},
\end{aligned}
$$

where $\mathbf{a}_{i \max }$ is the highest diagonal element of $\mathbf{M}_{i}$.

$$
\left(a_{i \min } / \mathbf{a}_{i \max }\right) x_{i}^{T} R_{i} x_{i} \leq\left(a_{i \min } / \mathbf{a}_{i \max }\right) x_{i}^{T} \mathbf{M}_{i} x_{i},
$$

where $a_{i \min }$ is the lowest diagonal element of $M_{i}$.

It is noted that

$$
\begin{aligned}
\left(a_{i \min } / \mathbf{a}_{i \max }\right) x_{i}^{T} \mathbf{M}_{i} x_{i} & \leq x_{i}^{T} M_{i} x_{i}, \\
\left(a_{i \min } / \mathbf{a}_{i \max }\right) x_{i}^{T} R_{i} x_{i} & \leq x_{i}^{T} M_{i} x_{i}, \\
x_{i}^{T} M_{i} x_{i} & \geq\left(a_{i \min } / a_{i \max }\right) x_{i}^{T} R_{i} x_{i}, \\
x_{i}^{T} M_{i} x_{i} & \geq 2 m_{i} x_{i}^{T} R_{i} x_{i},
\end{aligned}
$$

where

$$
m_{i}=1 /\left(2 \mathbf{a}_{i \max } / a_{i \min }\right) .
$$

$m_{i}$ is a real and positive number.

It is noted that in all the above cases, combining the above results with the results of appendices $\mathrm{A}-\mathrm{C}$ one gets

$$
\begin{aligned}
& \sum_{i=1}^{N} x_{i}^{T}\left(\left(1 / \lambda_{i}\right) R_{i} b_{i} b_{i}^{T} R_{i}\right) x_{i} \leq \sum_{i=1}^{N} x_{i}^{T}\left(A_{i i}^{T} R_{i}+R_{i} A_{i i}\right) x_{i} \\
& +\sum_{i=1}^{N} 2 m_{i} x_{i}^{T} R_{i} x_{i}+\sum_{i=1}^{N} x_{i}^{T} Q_{i} x_{i}
\end{aligned}
$$

\section{Appendix E.}

The inequality above in appendix $\mathrm{D}$ can be replaced by an equation, on replacing $R_{i}$ by another $n_{i} \times n_{i}$ real symmetric positive definite matrix $\mathbf{R}_{i}$,

$$
\begin{aligned}
& \sum_{i=1}^{N} x_{i}^{T}\left(\left(1 / \lambda_{i}\right) \mathbf{R}_{i} b_{i} b_{i}^{T} \mathbf{R}_{i}\right) x_{i}=\sum_{i=1}^{N} x_{i}^{T}\left(A_{i i}^{T} \mathbf{R}_{i}+\mathbf{R}_{i} A_{i i}\right) x_{i} \\
& \quad+\sum_{i=1}^{N} 2 m_{i} x_{i}^{T} \mathbf{R}_{i} x_{i}+\sum_{i=1}^{N} x_{i}^{T} Q_{i} x_{i}, \\
& x_{i}^{T}\left(\left(1 / \lambda_{i}\right) \mathbf{R}_{i} b_{i} b_{i}^{T} \mathbf{R}_{i}\right) x_{i}=x_{i}^{T}\left(A_{i i}^{T} \mathbf{R}_{i}+\mathbf{R}_{i} A_{i i}\right) x_{i}+2 m_{i} x_{i}^{T} \mathbf{R}_{i} x_{i}+x_{i}^{T} Q_{i} x_{i}, \\
& \left(1 / \lambda_{i}\right) \mathbf{R}_{i} b_{i} b_{i}^{T} \mathbf{R}_{i}=A_{i i}^{T} \mathbf{R}_{i}+\mathbf{R}_{i} A_{i i}+2 m_{i} \mathbf{R}_{i}+Q_{i}, \\
& \left(1 / \lambda_{i}\right) \mathbf{R}_{i} b_{i} b_{i}^{T} \mathbf{R}_{i}=\left(A_{i i}+m_{i} I\right)^{T} \mathbf{R}_{i}+\mathbf{R}_{i}\left(A_{i i}+m_{i} I\right)+Q_{i} .
\end{aligned}
$$




\section{References}

Kuo B C 1993 Automatic control systems P H I 6th edn, pp 222-225

Mahalanabis A K, Singh R 1980 On decentralised feedback stabilisation of large-scale interconnected systems. Int. J. Control 32: 115-126

Siljak D D, Vukcevic M B 1977 Decentrally stabilizable linear and bilinear large-scale systems. Int. J. Control 26: 289-305

Singh R 1970 Optimal feedback control of linear time-invariant system with quadratic criterion. Inst. Eng. (India). Electron. Telecommun. Div. 51: 52-55

Wang S H, Davison E J 1973 On the stabilisation of decentralised control systems. IEEE Trans. Autom. Control AC-18: 473-478 